\title{
La coparticipación provincial por salud en el Gran Buenos Aires: reflexiones en torno a la política local (2000-2005)
}

\author{
Provincial health funds distribution in the \\ Gran Buenos Aires: thoughts about \\ local policy (2000-2005)
}

Magdalena Chiara1 ${ }^{1}$ Mercedes Di Virgilio², Ana Ariovich ${ }^{3}$

\footnotetext{
${ }^{1}$ Licenciada en Ciencias Antropológicas, Universidad de Buenos Aires (UBA). Docente Investigadora del Instituto del Conurbano, Universidad Nacional de General Sarmiento (UNGS), Argentina. magdachiara@fibertel.com.ar

${ }^{2}$ Licenciada en Sociología. Doctora en Ciencias Sociales, Universidad de Buenos Aires (UBA). Investigadora del Instituto de Investigaciones Gino Germani, Facultad de Ciencias Sociales, Universidad de Buenos Aires (FCS-UBA), Argentina.

mdivirgilio@fibertel.com.ar

${ }^{3}$ Licenciada en Ciencias Antropológicas, Universidad de Buenos Aires (UBA). Becaria del Fondo para la Investigación Científica y Tecnológica (FONCYT), Universidad Nacional de General Sarmiento (UNGS), Argentina.

ariovich@ungs.edu.ar
}

RESUMEN En las últimas décadas el sector salud sufrió en la provincia de Buenos Aires un agudo proceso de descentralización de servicios hacia los municipios. El mismo, no estuvo acompañado por la definición de un modelo prestacional que instituyera los niveles a ser garantizados por cada una de las unidades territoriales menores. Este trabajo pone el foco en las consecuencias que han tenido en la política local los criterios de distribución de los fondos de coparticipación en "salud" desde el Estado provincial hacia los municipios. Interesa comprender cómo estos criterios incentivan, restringen y organizan la política sanitaria local dando lugar a una fragmentación que genera diferentes condiciones de ejercicio del derecho a la salud en cada espacio local. Como parte de un proyecto colectivo más general, el estudio busca reconstruir para los 24 municipios del Gran Buenos Aires las condiciones a nivel macro en que se desenvuelve la política local para detectar la existencia de "patrones típicos" frente a la crisis del año 2001, que permitan explicar la orientación de las estrategias locales.

PALABRAS CLAVE Políticas Públicas de Salud; Estrategias Locales; Equidad en Salud; Financiación de la Salud.

\begin{abstract}
In recent decades the health sector in the province of Buenos Aires has suffered an acute process of decentralization of services towards municipalities. This process was not accompanied by the definition of a benefit model establishing the level to be guaranteed in every smaller territorial unit. This work focuses on the impact that funds distribution criteria in "health" -from the provincial state to municipalities- have had in local policy. The objective of this study is to understand how this distribution encourages, restricts and organizes local health policy, generating a fragmentation that creates different conditions for exercising the right to Health in each local area. As part of a collective project, the study seeks to reconstruct the 24 municipalities of Gran Buenos Aires macro conditions of local policy in order to detect the existence of "typical patterns" during the crisis of 2001, which will help to explain the perspective of local strategies.
\end{abstract}

KEY WORDS Health Public Policy; Local Strategies; Equity in Health; Financing, Health. 


\section{PRESENTACIÓN}

En las últimas décadas el sector salud sufrió en la provincia de Buenos Aires un agudo proceso de descentralización de servicios hacia los municipios. Este proceso no se dio en el vacío, sino en una trama de reglas de juego que, por acción u omisión, constituyen incentivos o restricciones para la política de salud en el nivel local.

En el marco de estas reglas de juego, este trabajo pone el foco en las consecuencias que han tenido en la política local durante la crisis y en los años posteriores, los criterios de distribución de los fondos de coparticipación desde el Estado provincial hacia los municipios en lo referido al componente "salud" que, al operar como financiamiento del gasto ejecutado, se realiza en función de la producción del sistema. Interesa comprender cómo estos criterios incentivan, restringen y orientan la política sanitaria local.

Este estudio, basado en fuentes secundarias (datos epidemiológicos, de dotación de servicios de salud, de transferencias de recursos de la provincia hacia los municipios y recursos presupuestarios municipales), busca reconstruir, para los 24 municipios del Gran Buenos Aires, las condiciones a nivel macro en que se desenvuelve la política local, para detectar la existencia de patrones típicos frente a la crisis de 2001 y años posteriores, que organizan las estrategias locales en sus posibles relaciones con las características del territorio.

Se trata de una aproximación basada en datos cuantitativos, que requiere de la realización de estudios de caso en profundidad, parte de cuya tarea se está desarrollando en el contexto de un proyecto colectivo más general (a) del cual este trabajo forma parte.

\section{FRAGMENTACIÓN Y HETEROGENEIDAD: COORDENADAS DEL MAPA RESULTANTE DE LA DESCENTRALIZACIÓN EN LA PROVINCIA (b)}

La descentralización de los servicios de salud en la provincia de Buenos Aires fue resultado de procesos domésticos y de otros que expresaban las tendencias globales de las reformas de la política social. Entre los primeros, la finalización de la transferencia de los centros de salud provinciales a la órbita de los municipios fue una iniciativa importante que avanzó en la descentralización de las funciones relativas a la atención primaria de la salud en la esfera local. Otro fenómeno fue la decisión tomada en el año 1987 de incorporar a los recursos de coparticipación (c), las transferencias para el financiamiento de los servicios de salud a cargo de los municipios.

Entre aquellos procesos que expresaban las tendencias globales, puede señalarse la incidencia que tuvieron algunos programas con financiamiento externo, tanto en la focalización como en el esfuerzo por cambiar el modelo de gestión. Estas iniciativas, radicadas en el nivel nacional, tuvieron expresiones subnacionales en sus modos de implementación.

En consecuencia, en la provincia de Buenos Aires el subsector público de la salud se fue constituyendo (sobre la base de los principios del sistema federal) por un progresivo proceso de transferencia de funciones desde los niveles jurisdiccionales superiores (nacionales y provinciales) hacia los municipios, hacia efectores del sistema (como los hospitales) y hacia otros agentes. Inversamente, la reasunción del ejercicio de funciones por parte de la provincia en el sector (como la provincialización de algunos hospitales), ha tenido también gran capacidad de reorganización de las relaciones entre las jurisdicciones. Esta dinámica tuvo lugar de manera incremental sin la explicitación de los niveles de prestación y las funciones a cumplir por cada nivel.

A su vez, el primer nivel de atención ofrece en el Gran Buenos Aires una configuración particular: ha sido modelado sobre la base de la iniciativa de organizaciones sociales comunitarias (sociedades de fomento) que obtuvieron recursos de los gobiernos municipales y que fueron progresivamente ganando carácter estatal.

En la actualidad, la definición de la política de salud correspondiente al subsector público tiene por protagonistas, en el conjunto de la provincia, a las tres jurisdicciones (nacional, provincial y municipal) en la definición de regulaciones, en la gestión de recursos humanos y de servicios y en el diseño de políticas. El primer nivel de atención está compuesto por 1.676 centros de salud, de los cuales casi la totalidad (1.654) son municipales, al tiempo que el segundo nivel de 
atención dispone de 335 establecimientos hospitalarios que dependen de diferentes jurisdicciones (27.566 camas totales distribuidas en 2.122 nacionales, 12.831 provinciales y 12.613 municipales).

Otro rasgo particular de la provincia de Buenos Aires es la regionalización de la gestión (con atributos de desconcentración) que define un nivel intermedio de interlocución de actores y de acuerdos intergubernamentales (formales e informales) en los espacios de las Regiones Sanitarias, establecidas por la Ley 7.016 (3) y reglamentada por el Decreto 805/67 (4) de la legislación provincial.

Por otra parte, la importancia demográfica y la concentración de la pobreza son algunos de los atributos que hacen de la provincia en general, y del Gran Buenos Aires en especial, un territorio particular para la política sanitaria.

El Gran Buenos Aires (GBA) es el conjunto de los 24 municipios que constituyen la conurbación de la Ciudad Autónoma de Buenos Aires (d). Este agregado de municipios presenta características muy diferentes desde un punto de vista sociodemográfico, socioeconómico y sociosanitario.

La crisis económica, política y social, que alcanzó su punto más crítico en diciembre de 2001 (e), registró en el GBA los impactos más agudos. De acuerdo con los datos del Censo Nacional de Población, Hogares y Vivienda de 2001 (5), un $17,6 \%$ de la población del GBA tenía en ese año necesidades básicas insatisfechas. Sin embargo, los efectos de la crisis no fueron homogéneos: algunos municipios presentaban un $4 \%$ de su población con necesidades básicas insatisfechas y otros hasta un $26 \%$ en dicha situación.

Los datos del mismo censo ponen en evidencia también algunas otras heterogeneidades: mientras algunos municipios tenían un $27 \%$ de población sin cobertura de obra social y medicina privada (San Isidro, Vicente López), otros alcanzaban el 65\% (Moreno, Florencio Varela) (5).

Cabe destacar que, en un contexto de descentralización, estos atributos definen demandas sociales distintas y condiciones muy diferentes en la producción de la oferta, dando lugar a fuertes inequidades en el acceso a los servicios de salud. Tal como señaláramos anteriormente, este proceso no se dio en el vacío sino en una trama de reglas de juego -las denominadas condiciones del régimen de implementación (6)- que, por acción u omisión, constituyen incentivos o restricciones para la política de salud en el nivel local.

En suma, la descentralización de la salud en la provincia de Buenos Aires -idiosincrásicamente modelada- operó sobre un punto de partida institucionalmente débil y heterogéneo en los municipios y, en su devenir, fueron resolviéndose de modo distinto los problemas, configurando así espacios de producción de política sanitaria con atributos muy diferentes. El resultado final de estos procesos ha dado lugar en el nivel local a una brecha entre las nuevas responsabilidades asumidas por los municipios en el campo de la salud y el déficit de capacidad estatal para sostener su pleno ejercicio.

Los modos a través de los cuales se "cierra" la brecha modelan la política local de salud en lo referente a la atención, la gestión y el financiamiento, definiendo modalidades diferentes en el acceso, la intensidad y la cobertura de los servicios. Estos procesos no fueron homogéneos, sino que estuvieron condicionados -entre otros factores- por la incidencia que en cada territorio tuvieron los cambios contextuales ocurridos durante las últimas décadas y que encuentran en las crisis puntos de clivaje de suma relevancia. Estos quiebres han tenido consecuencias en el modelado de los problemas y de la agenda de gobierno local.

\section{LOS DISPOSITIVOS DE COORDINACIÓN DE LA POLÍTICA SANITARIA: PARTICULARIDADES DE LOS MECANISMOS DE DISTRIBUCIÓN DE RECURSOS COPARTICIPABLES}

Pasadas casi dos décadas, la descentralización en la provincia de Buenos Aires parece mostrar tanto los "beneficios" (al acercar los niveles de decisión a las preferencias de los ciudadanos) como los "riesgos" inherentes a la fuerzas centrífugas que moviliza (7).

En un contexto como el analizado, el desarrollo del potencial de autonomía, que la descentralización le confirió al nivel local, requiere de la existencia de mecanismos que garanticen la articulación del sistema en su conjunto, al menos por dos razones. En primer lugar, 
porque la población, en su tránsito por el sistema de salud, encuentra fronteras institucionales no evidentes, pudiendo generar economías externas de distinto tipo. En segundo lugar, porque esta tendencia a la autonomía puede estar dando lugar a distintos niveles de acceso al derecho a la salud.

Ahora bien, ¿cuáles son los dispositivos existentes de articulación del sistema?; ¿cuál es su campo de actuación?

Dado el alcance y los propósitos de este trabajo, "pasaremos en limpio" los mecanismos de articulación que, desde fuera de lo local, pueden incentivar, restringir y orientar la política local. Recuperando análisis precedentes (1), y poniendo el foco en la coyuntura en torno a la crisis 2001-2003, se advierte que la trama de relaciones en la cual se mueve la política local está vertebrada por distintos recursos (presupuestarios, organizacionales, simbólicos) entre los cuales mencionamos:

$\rightarrow$ los efectores (hospitales y centros de salud);

$\rightarrow$ los programas nacionales y provinciales y la capacidad para asignar responsabilidades en su gestión (centralizadas, desconcentradas o descentralizadas);

$\rightarrow$ la obra pública, particularmente en relación a la construcción de hospitales interzonales;

$\rightarrow$ las transferencias resultantes de la coparticipación así como otro tipo de transferencias no directamente vinculadas al sector.

Del conjunto de mecanismos existentes, interesa en este trabajo poner el foco en los incentivos y las restricciones que imponen los mecanismos de distribución secundaria de la coparticipación (f).

El cálculo de los mecanismos de distribución secundaria de la coparticipación -en especial aquellos que tienen relación directa con las prestaciones de salud- al operar como financiamiento del gasto ejecutado, se realiza en función de la producción del sistema; se trata del único sistema en el país que tiene un distribuidor asociado a salud. Este sistema fue adoptado en la provincia en 1987 con el objetivo de dar respuesta a los problemas financieros que acumulaban los municipios como consecuencia de la incorporación de los servicios transferidos.
La Ley provincial 10.820 (8) de 1989 fijó los criterios de asignación secundaria de los recursos; esta Ley mantuvo los criterios establecidos en la Ley provincial 10.559 (9) y agregó otros destinados a contemplar las diferencias de productividad entre los establecimientos de salud.

Según larussi (10), la Ley 10.820 no plantea una transferencia de recursos como incentivo para guiar una política sanitaria en el ámbito local, sino un sistema de transferencias de carácter devolutivo: se recaudan los impuestos y se devuelven a las instancias responsables de los servicios. Cabe señalar que esta norma compromete un $37,13 \%$ del total de la masa coparticipable, y lo distribuye sobre la base de los siguientes criterios:

1. $35 \%$ en proporción directa al número de camas, perfil de complejidad y porcentaje ocupacional de camas de los establecimientos con internación de cada partido.

2. $25 \%$ en proporción directa al número de consultas médicas registradas en los establecimientos -con o sin internación- de cada partido.

3. $10 \%$ en proporción directa al número de egresos registrados en los establecimientos con internación en cada partido.

4. $20 \%$ en proporción directa al número de pacientes-días registrados en los establecimientos con internación de cada partido.

5. $10 \%$ en forma proporcional al número de establecimientos sin internación en cada partido.

Tal como están definidos en la legislación vigente, estos criterios presentan diversos problemas que han sido analizados en distintos trabajos (10-13):

1. La existencia de problemas en el financiamiento por insuficiencia de la masa coparticipable, atributo del sistema que afecta a todos los municipios por igual.

2. El hecho de que un régimen especial (el de salud) "sea incorporado dentro de un régimen general de coparticipación que persigue otros objetivos" (13), ya que la masa de coparticipación puede aumentar o disminuir según los ciclos macroeconómicos y no alcanzar -en algunos casos- a cubrir los gastos derivados de la prestación de los servicios (g). 
3. La fórmula actual introduce diferencias en el financiamiento entre municipios y distorsiones en la forma en la cual los municipios asignan los recursos para salud (algunas distorsiones son originadas por el uso de la máxima complejidad municipal para ponderar las camas ocupadas).

4. La asignación de porcentajes fijos a las variables integrantes de la fórmula implica que el pago unitario por variable será menor toda vez que la producción crezca a mayor tasa que la masa coparticipable. Este fenómeno se ve claramente en el valor transferido por consulta que es cada vez menor.

5. Los incentivos implícitos en los indicadores instituidos desde la ley, parecen haberse profundizado a partir de una Resolución del Ministerio de Economía de la Provincia de Buenos Aires (Res. 198/89) en el año 1989, la cual determina que el "perfil de complejidad" es la máxima complejidad existente en el municipio. Dada esta regla de juego, esta variable genera un rápido impacto en el aumento de la coparticipación, modificando los valores a transferir en la totalidad de las camas municipales (sean agudas o crónicas) de modo tal que las camas ocupadas de la misma complejidad resultan valorizadas de manera diferente en distintos municipios.

6. Sobrefinanciamientos y subfinanciamientos en los distintos componentes, resultantes de la rigidez de la fórmula y de la aplicación de un $35 \%$ por el perfil de complejidad (10).

7. La existencia de problemas de sobredeclaración de prestaciones, derivaciones de patologías costosas, sobreequipamiento y estrategias de elevación de la complejidad.

Por último, y en parte como resultado de los problemas antes enunciados, estos mecanismos tienen consecuencias en la inequidad. Volveremos sobre este aspecto más adelante.

Tal como plantea larussi (10), el sistema se originó en la necesidad de dar respuesta al problema del financiamiento de los servicios transferidos y presenta un carácter devolutivo, a la vez que no busca orientar la definición de políticas locales. Sin embargo, en un contexto de desarrollo progresivo de los sistemas de salud en el nivel local a lo largo de la década pasada, y ante la ausencia de una política de salud acordada entre los municipios y la provincia, las reglas de juego presentes en la distribución secundaria de la coparticipación se convirtieron en incentivos y restricciones al momento de definir una política local y su financiamiento, o viceversa.

Cabe destacar que el resultado de la distribución de los fondos de coparticipación por salud representa en sus valores agregados -según estimaciones del año 2005- el 70\% del gasto total en salud (776 millones de pesos corrientes), mientras que el $30 \%$ restante (322 millones de pesos corrientes) del financiamiento de los servicios bajo jurisdicción municipal son financiados con fondos municipales derivados de tasas y otros ingresos (10). Estos valores, en el nivel de los datos agregados, encierran situaciones muy diversas; mientras en algunos municipios del interior de la provincia, el financiamiento de la coparticipación supera el $100 \%$ de lo gastado en salud, para los municipios del Gran Buenos Aires, los porcentajes de financiamiento del gasto municipal en salud se reducen a alrededor del $50 \%$. En esta región, también este valor promedio comprende situaciones diferentes cuya lógica buscamos indagar en este trabajo: mientras algunos municipios financian alrededor del $20 \%$ de su gasto en salud con las transferencias resultantes de la coparticipación (Tres de Febrero, San Fernando y Ezeiza), otros Ilegan a financiar alrededor del $80 \%$ (San Miguel y Malvinas Argentinas) (h).

\section{CONDICIONALIDADES O ESTRATEGIAS: ¿DISTINTOS PATRONES EN EL NIVEL LOCAL?}

Dada la situación de autonomía generada posteriormente a la descentralización, cabe preguntarse en torno a qué estrategias se han organizado los gobiernos locales en función de optimizar (o no) la captación de los recursos, y cuáles han sido sus consecuencias.

A partir del análisis de la serie 20012005 correspondiente a las transferencias realizadas por el Estado provincial a los municipios del GBA, en función del distribuidor salud de la coparticipación, y en contrapunto con otras variables (vinculadas a las condiciones de vida de 
la población, situación sanitaria y organización del sistema), los resultados que aquí se presentan proveen pistas para pensar cuál ha sido la relación entre los recursos transferidos y la demanda potencial, cuál ha sido el impacto de la crisis 2001-2003 en estas transferencias según los diferentes municipios de la región, y si existen condiciones de partida en los municipios que permitan explicar estrategias diferenciales.

\section{RELACIÓN ENTRE LOS RECURSOS TRANSFERIDOS Y LA DEMANDA POTENCIAL}

Debido a la importancia que tiene la presencia de establecimientos con internación bajo la órbita municipal, y considerando la hete- rogeneidad que presentan los municipios del GBA en ese sentido, consideramos en el análisis solo aquellos componentes de la coparticipación que ponderan aspectos vinculados a la atención ambulatoria (consultas y establecimientos sin internación) correspondientes al año 2005, período en que consideramos pasada la crisis (i).

Teniendo en cuenta ese recorte, los valores en pesos de la coparticipación en salud (componente consultas más establecimientos sin internación) por el número total de habitantes, ponen en evidencia algunas situaciones particulares que dan cuenta de la existencia de ciertas brechas entre municipios. Mientras diez municipios presentan valores por debajo de los 14,99 pesos corrientes (Gral. San Martín, Tres de Febrero, Morón, Ituzaingó, Moreno, Merlo, MÁS ESTABLECIMIENTOS SIN INTERNACIÓN Y NÚMERO TOTAL DE HABITANTES. MUNICIPIOS DEL GRAN BUENOS AIRES, ARGENTINA. 2005.

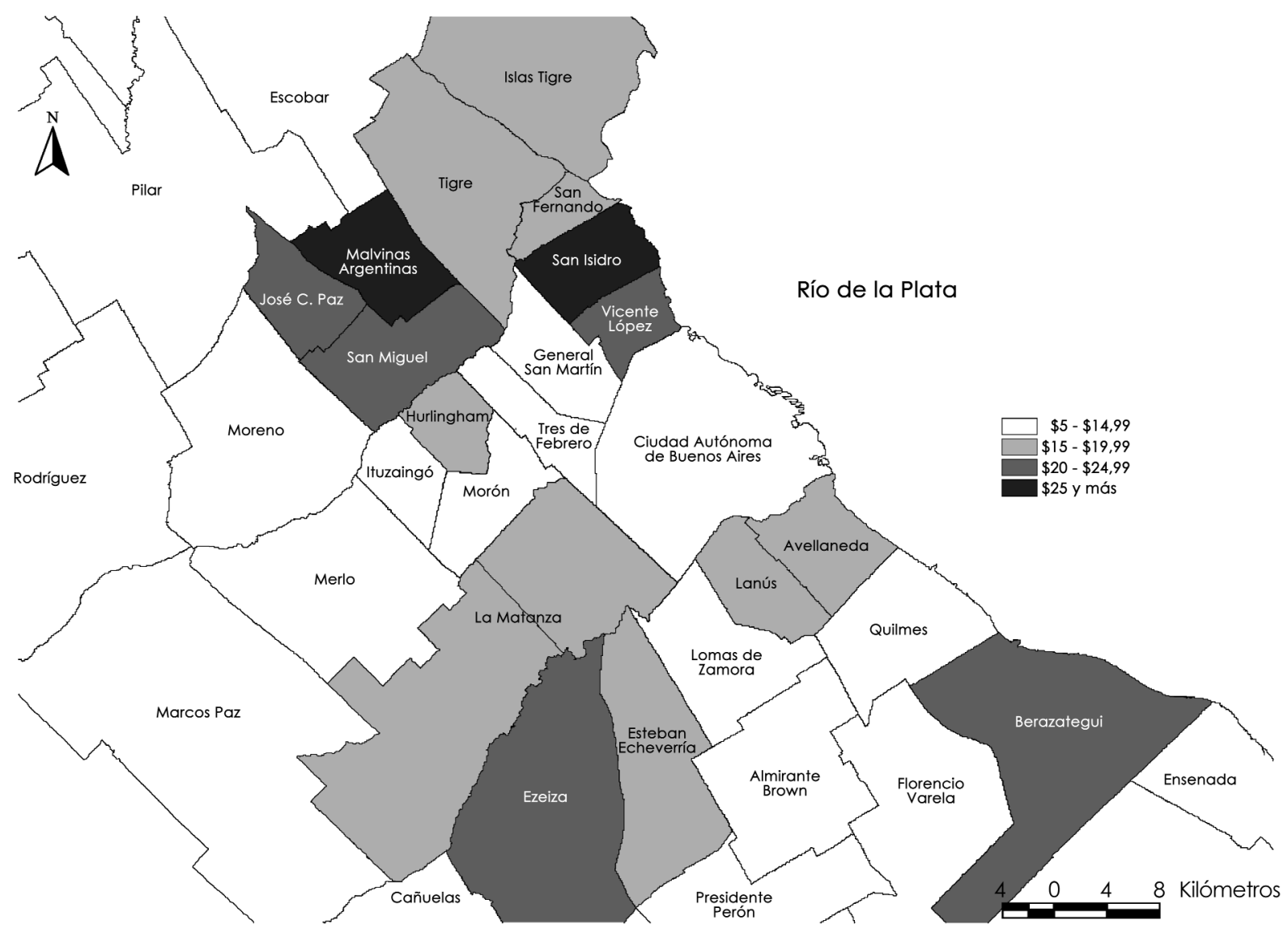

Fuente: Elaboración propia en base a Chiara, Di Virgilio y Miraglia (2). 
Lomas de Zamora, Quilmes, Almirante Brown, Florencio Varela), en otros casos, los valores superan los 25 pesos corrientes para el mismo indicador (municipios de Malvinas Argentinas y San Isidro) (ver Figura 1). No obstante el esfuerzo puesto aquí en aislar la incidencia de la presencia o ausencia de hospital bajo jurisdicción municipal, cabe destacar que el componente "consultas" está todavía influido por este factor. En este caso, las transferencias promedio más elevadas se encuentran en aquellos municipios que han apostado a tener establecimientos con internación bajo su jurisdicción.

Estas brechas profundizan la hipótesis de la heterogeneidad de los sistemas locales (en dotación y alcance de la red de servicios disponibles) a la que hacíamos referencia, fenómeno que no parece relacionarse -en todos los casos- con la demanda potencial de cada partido.

La mirada que estamos proponiendo supone la existencia de sistemas organizados universalmente. Sin embargo, la demanda sobre el subsector público parece variar según sea la cobertura alternativa con que cuente la población y sus niveles de bienestar.

El análisis realizado más arriba mostraba las diferencias que existen entre los municipios del GBA en el porcentaje de población sin cobertura de obra social y medicina privada. Este dato pone en evidencia demandas muy diferentes sobre el subsector público en unos casos respecto de otros. En ese sentido, otra aproximación posible a la relación entre "transferencias" y "demanda" es la que vincula los componentes seleccionados de la

Figura 2. DISTRIBUCIÓN DE LA COPARTICIPACIÓN EN SALUD. RELACIÓN ENTRE COMPONENTE CONSULTAS MÁS ESTABLECIMIENTOS SIN INTERNACIÓN Y NÚMERO TOTAL DE HABITANTES SIN COBERTURA DE OBRA SOCIAL O DE MEDICINA PRIVADA. MUNICIPIOS DEL GRAN BUENOS AIRES, ARGENTINA. 2005.

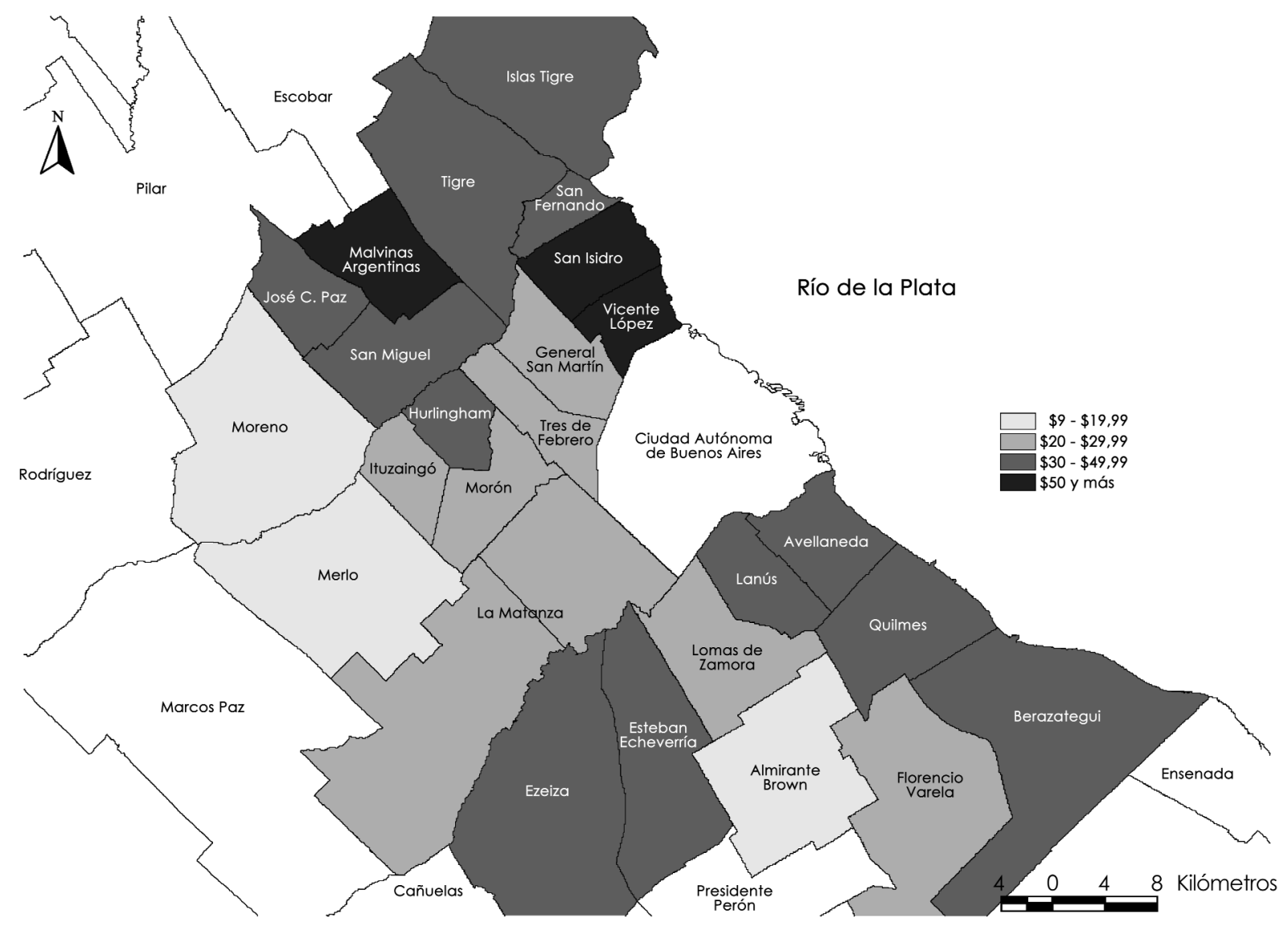

Fuente: Elaboración propia en base a Chiara, Di Virgilio y Miraglia (2). 
Figura 3. TENDENCIA TEMPORAL DE LAS TRANSFERENCIAS A LOS MUNICIPIOS DEL GRAN BUENOS AIRES, POR LA COPARTICIPACIÓN EN SALUD, A PESOS CORRIENTES Y A PESOS CONSTANTES (AJUSTADOS POR EL ÍNDICE DE PRECIOS MAYORISTAS). ARGENTINA, 2000-2005.

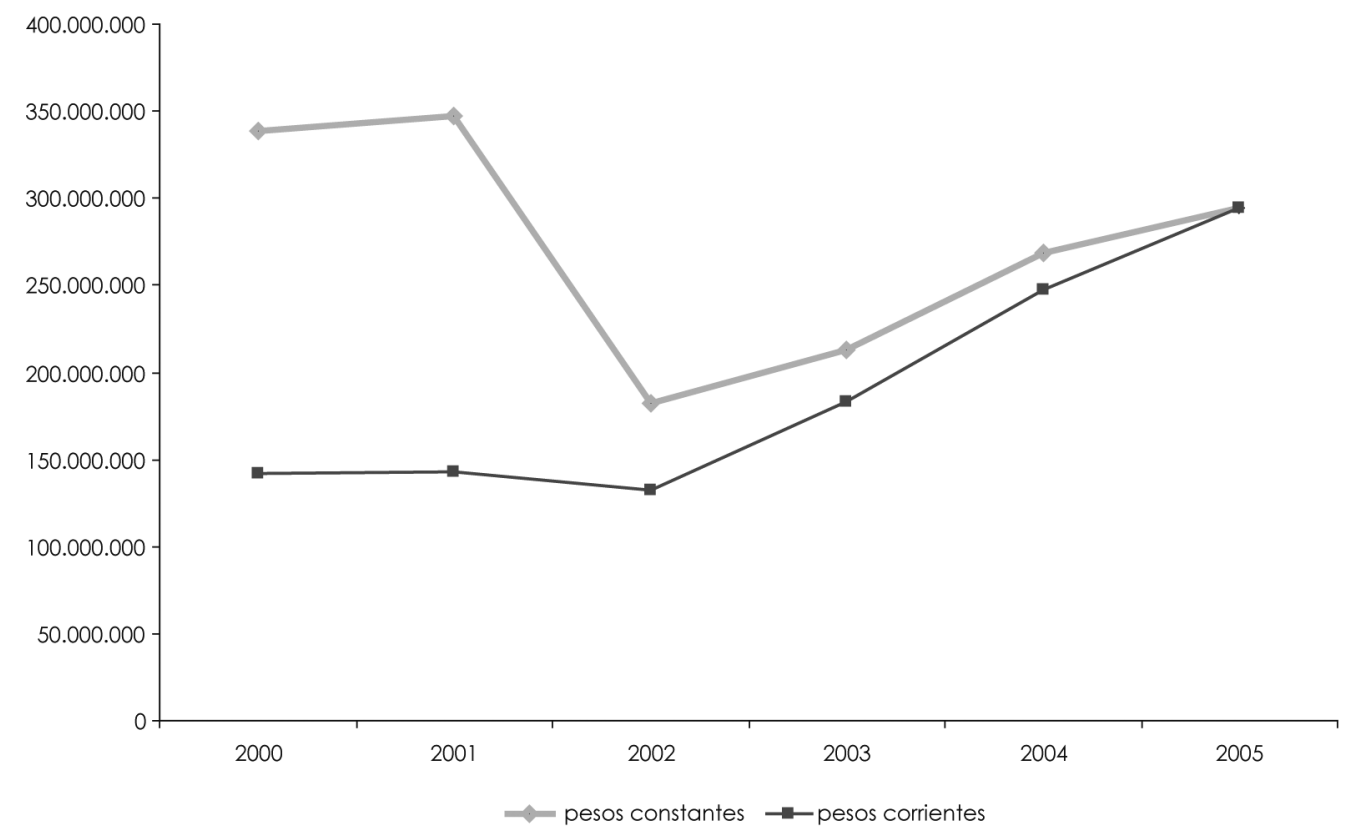

Fuente: Elaboración propia en base a datos suministrados por la Subsecretaría de Planificación de la Salud del Ministerio de Salud de la Provincia de Buenos Aires e Índice de precios mayoristas del Instituto Nacional de Estadísticas y Censos (14).

coparticipación y la población sin cobertura de obra social y medicina privada.

Las transferencias para el año 2005, medidas en pesos coparticipados (componente consultas más establecimientos sin internación) por habitante sin cobertura de obra social y medicina privada, presentan los mayores valores en los municipios de Malvinas Argentinas, Vicente López y San Isidro, donde superan los 50 pesos corrientes por habitante sin cobertura. Esto último pone en evidencia que aquellos partidos del GBA que presentan menores porcentajes de población sin obra social y medicina privada y mejores indicadores socioeconómicos, como Vicente López y San Isidro, reciben una alta coparticipación en salud.

Este indicador (pesos coparticipados -componente consultas más establecimientos sin internación- por habitante sin cobertura de obra social y medicina privada) también permite establecer diferencias internas en el grupo de los diez municipios que contemplan los valores más bajos en el análisis de transferencias recibidas per cápita sobre población total, realizado precedentemente. En este sentido, los municipios de Almirante Brown, Merlo y Moreno (que integran el mencionado grupo) presentan, de acuerdo al nuevo indicador, una coparticipación inferior a los 19,99 pesos por habitante sin cobertura de obra social o de medicina privada. Contrariamente, Quilmes -también perteneciente al grupo que contempla los valores más bajos de coparticipación por habitante total- se ubica entre un grupo de municipios cuya coparticipación en pesos por habitante sin cobertura es mucho más alta (con valores corrientes que oscilan entre los 30 y los 49,99 pesos) (ver Figura 2). Esta diferenciación parece estar definida desde variables sociales y económicas.

Es decir, si consideramos que las transferencias provinciales son un reflejo de la dotación y capacidad de producción que tienen los 
sistemas, el análisis realizado aquí pone sin duda en evidencia la diversidad de ofertas que comprenden los distintos sistemas locales.

\section{LA CRISIS 2001-2003 COMO PUNTO DE INFLEXIÓN}

Ahora bien, dado el interés en indagar cómo estas reglas de juego incentivan, limitan u orientan las políticas locales, cabe interrogarse sobre la evolución de las transferencias y sus consecuencias.

Una primera aproximación apunta a conocer cómo impactó la crisis 2001-2003 en las transferencias desde la provincia a los municipios del Gran Buenos Aires.

El análisis de los recursos totales en concepto de coparticipación en salud que reciben los municipios en la provincia de Buenos Aires muestra una tendencia hacia una baja, bastante generalizada para los 24 municipios de la región en el período 2000-2002, y una recuperación a partir de los años 2003-2004, momento en el que comienza a verificarse el impacto de la recuperación de la economía en la recaudación. A modo indicativo, podemos afirmar que en pesos constantes la masa coparticipable en concepto de salud no parece recuperar el valor que tenía antes del inicio de la crisis (ver Figura 3); en tanto nuestro interés radica en indagar en las estrategias seguidas, referimos a series en pesos corrientes conscientes de la pérdida del valor de compra, en particular con relación a insumos y equipamientos.

Esta dinámica pone en evidencia -en dos momentos diferentes- lo planteado por otros autores (13) acerca del carácter cíclico de este tipo de transferencias y los problemas, que en términos de sustentabilidad, puede generar para las estrategias locales.

Hay que destacar que la estructura de estas transferencias destinadas al financiamiento de la salud, se mantiene estable a lo largo del período 2000-2002, con algunas situaciones particulares. Mientras la mayor parte de los municipios crece en pesos corrientes entre un $50 \%$ y el $130 \%$, un solo municipio desciende un $42 \%$ (Florencio Varela), y otros cuatro crecen en valores que oscilan entre $160 \%$ y $500 \%$ con mucha dispersión entre sí: se trata de los municipios de Ezeiza, José C. Paz, Hurlingham y Malvinas Argentinas.
Si el año 2002 es un punto de inflexión para los valores agregados de transferencias en la región, cabe interrogarse acerca de los comportamientos diferenciales de los municipios para ese primer momento en que se reducen las transferencias.

Aunque se trató de un proceso generalizado para los municipios del GBA, hay un conjunto de municipios que -ya en este período- no redujeron (en pesos corrientes) los fondos recibidos por esta vía: los municipios de Berazategui, Hurlingham y José C. Paz. Estos municipios están levemente por encima del valor (siempre en pesos corrientes) correspondiente al año 2000, mientras que otro grupo de municipios presentan una diferencia positiva importante respecto del conjunto: los municipios de Ezeiza y Malvinas Argentinas. Los municipios de San Fernando y Tigre parecen haber estado afectados más particularmente en oportunidad de esta crisis.

Al salir de la crisis de los años 20012003 y como consecuencia de los impactos de la recuperación de la economía en la recaudación, tiene lugar un incremento de los fondos recibidos por los municipios (siempre a valores corrientes). A excepción del municipio de Florencio Varela (que provincializa su hospital), el resto recupera entre el $70 \%$ y el $150 \%$ de los recursos en pesos corrientes, respecto del año 2002. Por encima de ellos, aunque bastante cerca, se encuentran los municipios de Tigre y José C. Paz (157\% y $170 \%$ respectivamente).

Una situación muy particular es la que presenta el municipio de Malvinas Argentinas que, como vimos, pudo atravesar la crisis "ganando" recursos (al menos respecto del resto de los municipios) y que muestra, en la "salida" de la crisis (período 2003-2004), mayores niveles de captación de recursos a través del aprovechamiento de las reglas de juego de la coparticipación (con un incremento en pesos corrientes del $362 \%$ respecto de los valores del año 2002). Aunque bastante por debajo, se encuentra también el municipio de Hurlingham con un crecimiento del 225\% (ver Figura 4).

Si el análisis de la evolución del gasto pone en evidencia impactos distintos en la disponibilidad de recursos en el nivel local para el desarrollo de política sanitaria, la aproximación a las estructuras de distribución "punta a punta" de los 


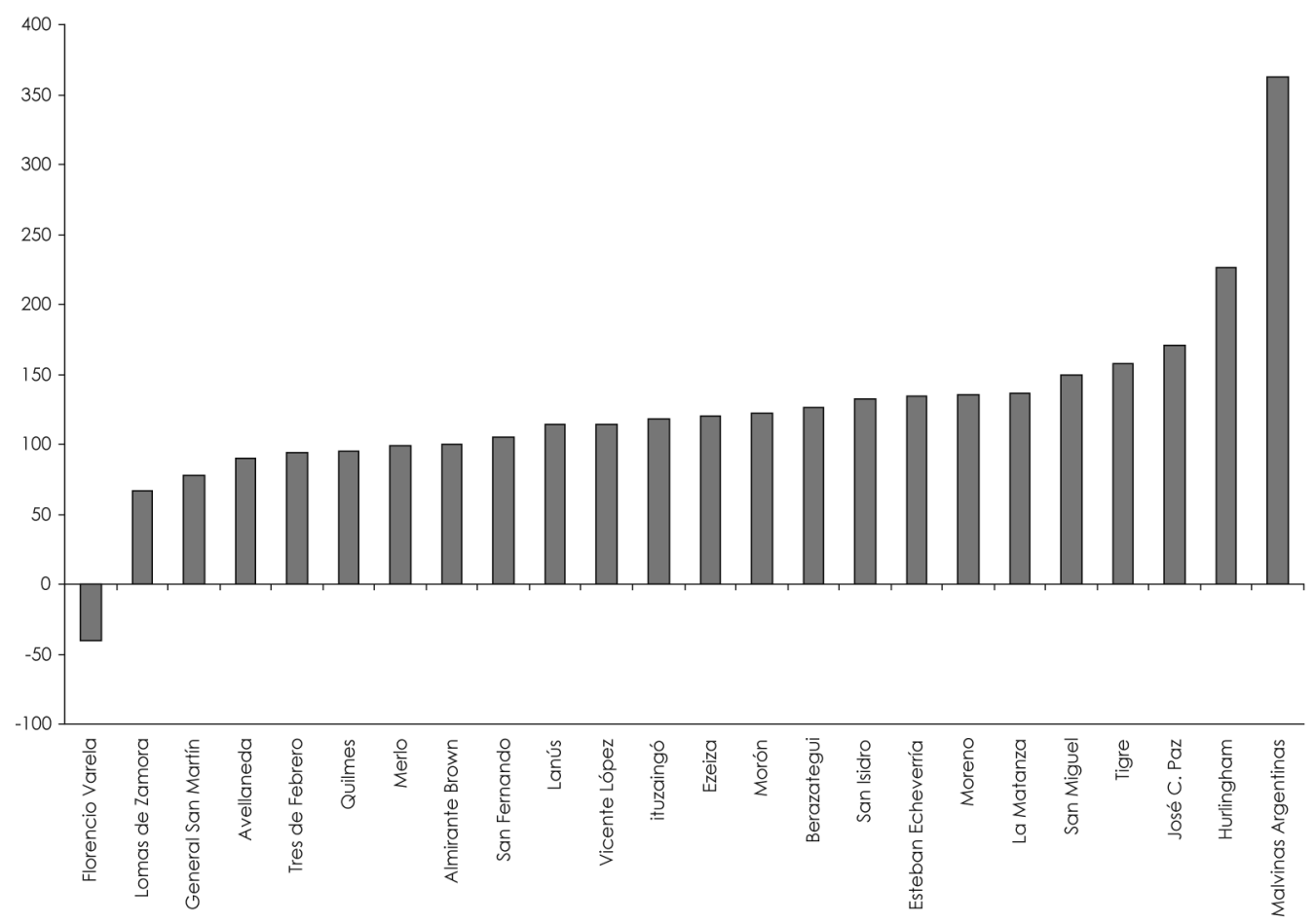

Fuente: Elaboración propia en base a datos suministrados por la Subsecretaría de Planificación de la Salud del Ministerio de Salud de la Provincia de Buenos Aires.

recursos, en el período 2000-2005, muestra pocos cambios en casi la totalidad de los municipios de la región, a excepción de los incrementos en el municipio de Malvinas Argentinas (que pasa del $4,62 \%$ a concentrar el $13,49 \%$ de los recursos coparticipado) y, mucho más lejos, en el municipio de La Matanza (que pasa del 14,3\% al $15,60 \%)$. Cabe destacar como contrapartida, el descenso en el municipio de Florencio Varela (que va del $8,04 \%$ al 2,24\%) y en el municipio de San Martín (que va del 6,75\% al 4,88\%) (ver Figura 5).

El análisis realizado hasta aquí muestra el carácter cíclico de los recursos recibidos por los municipios en materia de coparticipación. Este atributo se expresa, en este período, en una dinámica a través de la cual descienden los recursos (siempre a valores corrientes) en el período 2000-2002, y crecen a partir del 2002, como resultado de los impactos de la recuperación de la economía en la recaudación impositiva.

Esta dinámica está regida por reglas de juego que, aunque homogéneas para el conjunto de los municipios, dan lugar a situaciones diferenciales. Por una parte, la circunstancia particular de tres municipios (Florencio Varela, Malvinas Argentinas y Hurligham) que da cuenta de estrategias diferenciadas de incorporación de estas reglas de juego a la política local, sea por la vía de su aprovechamiento en la generación de ingresos o a través del alivio del gasto. Por otra, la situación de los restantes municipios del GBA que responden (con muy leves variaciones) a las tendencias agregadas.

Siguiendo el recorte regional de este trabajo, y buscando interrogar a las particularidades, indagaremos en las condiciones bajo las cuales se gestaron y desarrollaron estas estrategias. 


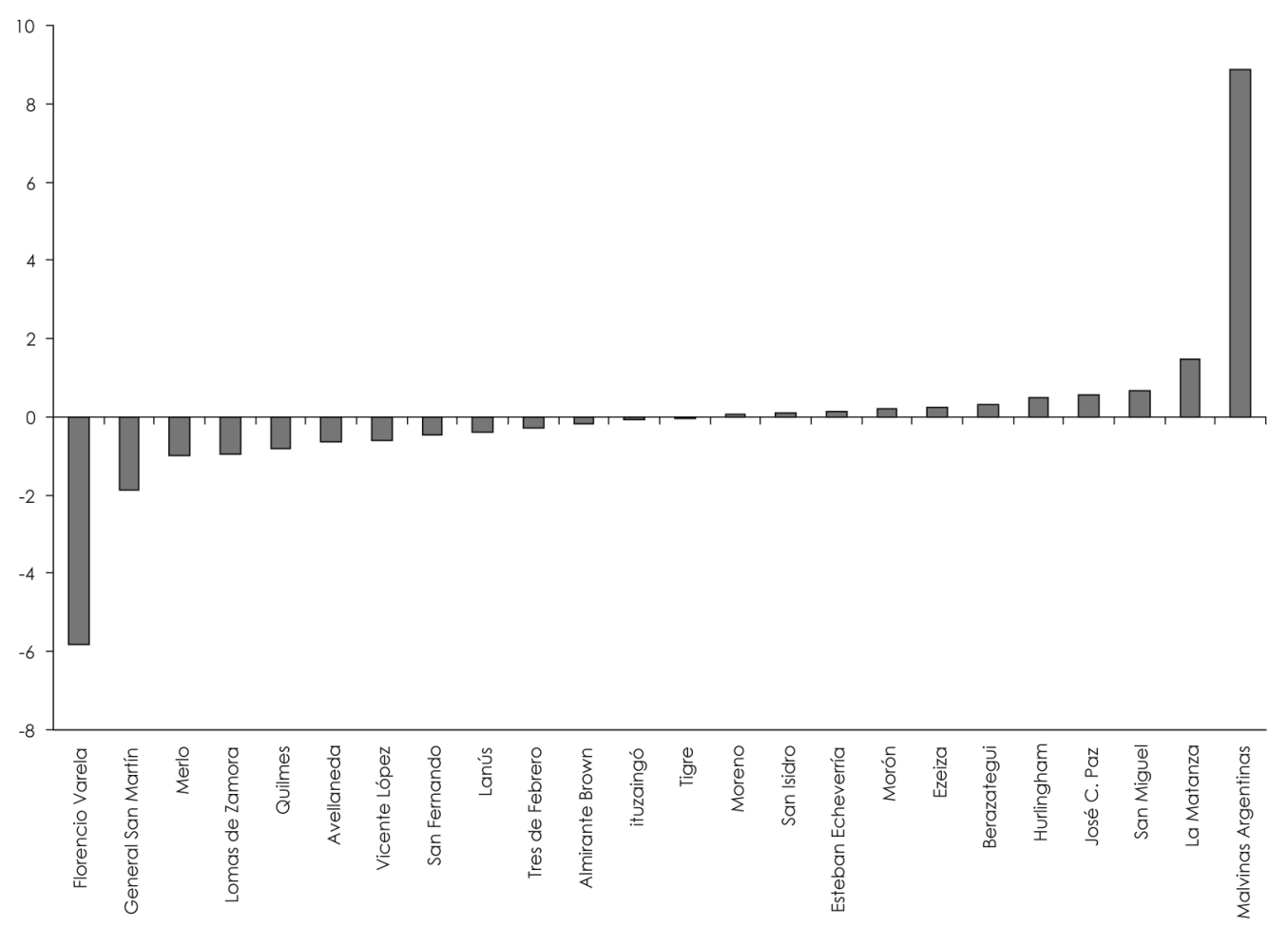

Fuente: Elaboración propia en base a datos suministrados por la Subsecretaría de Planificación de la Salud del Ministerio de Salud de la Provincia de Buenos Aires.

\section{LAS CONDICIONES DESDE LAS QUE SE GESTAN LAS ESTRATEGIAS LOCALES: ATRIBUTOS AGREGADOS DE LA DEMANDA Y LA DOTACIÓN DEL SISTEMA}

Una primera cuestión que interesa preguntarse es si la demanda de la población incide en el "aprovechamiento" de las reglas de juego del sistema desde la política local.

La situación de pobreza -medida por porcentaje de población con necesidades básicas insatisfechas (NBI) - y el nivel de cobertura de otros subsectores (medido en porcentaje de población sin cobertura de obra social y medicina privada) no parecen marcar diferencias en las condiciones de partida de los municipios para optar por una u otra estrategia con relación a estas reglas de juego. Los municipios que adoptan estrategias más extremas (provincializar el hospital en el caso de Florencio Varela o ampliar la dotación de servicios en el caso de Malvinas Argentinas) cuentan de acuerdo con datos del Censo Nacional de Población, Hogares y
Vivienda de 2001 (5), con condiciones críticas en términos de pobreza por NBI $(22,9 \%$ y $30,4 \%$, respectivamente) y también enfrentan el desafío de vastos sectores de su población sin otra cobertura que la del subsector público $(65,3 \%$ y $58,4 \%$ respectivamente).

La mayor parte de los municipios parece haber apostado por no cambiar la estrategia, limitándose a recibir las externalidades de un ciclo económico favorable que, considerando el incremento de los precios de insumos y equipamiento expresado en el ajuste por inflación (estimado más arriba), les permitiría apenas recuperar los valores previos a la crisis.

Si la primera línea de reflexión buscaba atributos que explicaran la adopción de una u otra estrategia desde las condiciones de vida de la población y su correlato en la demanda, la segunda interroga el origen de las estrategias desde el análisis de la dotación de efectores críticos disponibles a partir del incremento de la cantidad de camas. 
El caso que se está destacando por la apuesta al incremento de los ingresos, el municipio de Malvinas Argentinas (j), comienza a transitar este período con 187 camas municipales (como única dotación pública) y sale en el 2005 con 388 camas bajo jurisdicción municipal. Sin duda, esta decisión permitió internalizar en el sistema local (y en la gobernabilidad de las decisiones municipales) las necesidades de derivaciones de "casi" la totalidad de las patologías (k). Además, debe destacarse que la aceleración en la creación de camas en este municipio se dio entre los años 2002-2003, registrando un 56,7\% de crecimiento; este valor es particularmente significativo si se tiene en cuenta que para el conjunto del GBA las camas municipales sufrieron una variación de solo un 9,5\%. La apuesta por la creación de camas por parte de este municipio se desacelera en el período 2003-2005, sufriendo la cantidad de camas una variación $(7,2 \%)$ algo más cercana al promedio provincial (4\%) (15). No obstante, cabe destacar que en este período sigue creciendo diferencialmente la captación de recursos vía coparticipación en salud (como ya fue mencionado anteriormente), lo cual permite hipotetizar en torno a una estrategia más compleja consistente en: aumentar primero las camas y, posteriormente, la complejidad y las consultas.

En el otro extremo, el caso que apuesta a la reducción del gasto, nos encontramos con el municipio de Florencio Varela. Este municipio ingresa con 216 camas municipales totales y finaliza en el año 2005 con 233 camas, 185 provinciales y solo 48 municipales.

El análisis realizado hasta aquí es general y permite identificar casos típicos que pueden ser analizados en profundidad con otro tipo de aproximación. En el contexto del proyecto de investigación en el que se realiza este trabajo, se está avanzando en el estudio de tres casos: el municipio de Tigre (que presenta una situación estable en términos de su participación en el conjunto de las transferencias del GBA); el municipio de San Fernando (que ve reducida su participación en esa masa coparticipada regional); y, por último, el municipio de Malvinas Argentinas (que "gana" participación en el conjunto de la distribución de recursos en la región en este período). Se trata, en los dos primeros casos, de municipios que tienen muy pocas camas bajo jurisdicción municipal, mientras que Malvinas Argentinas tiene la totalidad de las camas bajo su jurisdicción.

Las características de los "equipos de cambio" (I) presentes en cada una de las Secretarías de Salud municipales, puede ser una pista para explicar las diferencias, en tanto la pertenencia a un partido político determinado no parece ser un factor explicativo. Este es el punto en que una aproximación como esta deja paso a otro tipo de estudio: cualitativo e histórico, que triangule fuentes y busque los factores que permitan explicar las causas de estas diferencias.

Las particularidades del entramado de actores parecen ser también un factor clave. La fortaleza o debilidad de los actores gremiales puede ser una variable importante a atender en la posibilidad de transitar uno $u$ otro camino. El incremento de camas y su habilitación para poder acreditarse de cara a la coparticipación supone, desde el punto de vista de los recursos, varios requisitos, a saber:

a) Disponer de recursos materiales o financieros o movilizar programas supralocales para invertir en la construcción de los establecimientos y su posterior equipamiento.

b) Disponer de capital político y capacidad de acción para disputar en los espacios institucionales el alta de camas y la acreditación de la complejidad de los establecimientos.

c) Contar con dispositivos de contratación de recursos humanos que permitan organizar en tiempos cortos los equipos médicos y no médicos para los efectores y servicios que se incorporan al sistema.

Este último requisito, que podría suponerse asociado a las estrategias orientadas al aprovechamiento de las condiciones que impone el régimen de coparticipación, implica además la necesidad de contar con amplios márgenes de gobernabilidad y autonomía de la acción gubernamental, especialmente con relación a las organizaciones gremiales. Desde este punto de vista, la precarización en las formas de incorporación de los profesionales médicos y no médicos que conforman los equipos de salud, parece ser resultado no solo de la urgencia por conformar equipos, sino también de la inestabilidad de los ingresos que pueden incrementarse 
al calor de la recuperación del ciclo económico, pero también descender.

\section{UNA APROXIMACIÓN FINAL SOBRE SUS RESULTADOS}

Hasta aquí hemos intentando discriminar, en el conjunto del GBA, situaciones diferenciales en relación a la coparticipación en salud. Sin embargo, quedan planteadas una serie de cuestiones que están siendo indagadas, desde nuestro proyecto más general, en los casos seleccionados.

Aunque advirtiendo las limitaciones existentes para poder dar cuenta de los avances, nos interesa marcar algunas líneas de análisis que permitirían realizar una valoración de las distintas apuestas.
Si bien la reducción de la mortalidad no es una función lineal de las transferencias y su elasticidad depende del desempeño de la tasa en una serie de tiempo -a menor mortalidad menor elasticidad-, parece importante contrastar el análisis con algún indicador disponible que dé cuenta de la situación en salud de la población. En este marco, si comparamos la variación interanual (2001-2005) que han sufrido estas transferencias en concepto de coparticipación en salud con aquella que sufrió la mortalidad infantil en el período analizado (2001-2005), podemos advertir que las apuestas no estuvieron asociadas a avances con relación a esta problemática, sea por dificultades inherentes a su modificación o porque no la priorizaron.

Los municipios que más variaron en los recursos coparticipados en salud, presentan muy leves variaciones en este indicador: Hurlingham

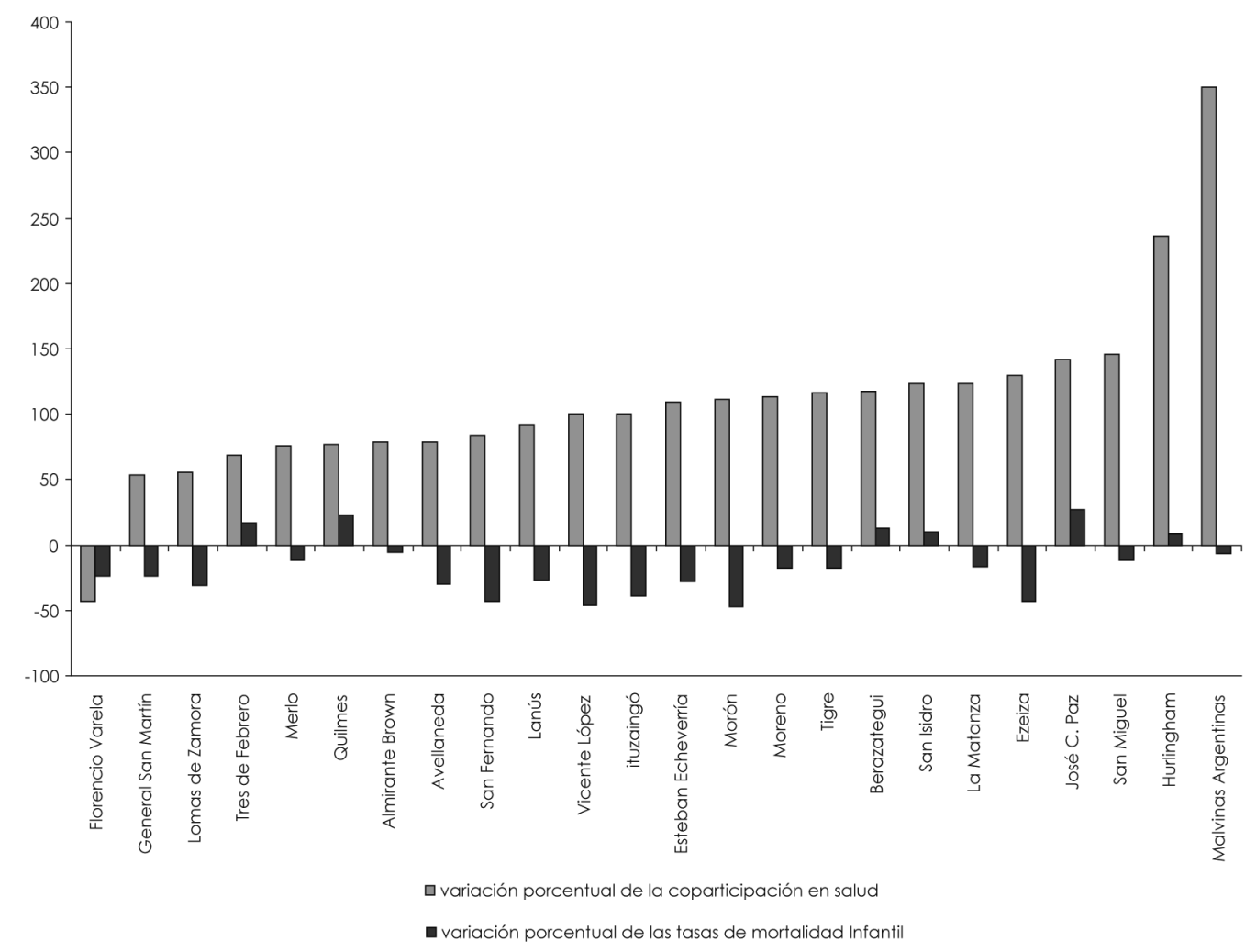

Fuente: Elaboración propia en base a datos de coparticipación suministrados por la Subsecretaría de Planificación de la Salud y de mortalidad infantil de la Dirección de Información Sistematizada del Ministerio de Salud de la Provincia de Buenos Aires (17). 
incrementa los recursos recibidos por este componente en $236,8 \%$, mientras que la tasa de mortalidad infantil sube del 11 por mil en el 2001 al 12 por mil en el 2005; en el municipio de Malvinas Argentinas, la variación de las transferencias por salud son de un $350 \%$ y la mortalidad infantil se reduce levemente del 16,9 por mil al 15,8 por mil (ver figura 6).

La apuesta a la reducción de la presión presupuestaria vía la provincialización del hospital coincidió en muchos casos con un mejor desempeño en términos de la reducción de la mortalidad infantil (es el caso de Florencio Varela). Aquellos que se mantuvieron en niveles más o menos homogéneos (con crecimientos entre el $50 \%$ y el $160 \%$ ) también redujeron la mortalidad infantil, a excepción de Tres de Febrero, Quilmes, Berazategui, San Isidro y José C. Paz.

Estos hallazgos nos invitan a reflexionar, entonces, sobre la relación que existe entre las estrategias diferenciales que expresan los cambios en las transferencias y la situación de salud de la población (medida aquí y a modo de ejemplo) en términos de la variación de la mortalidad infantil. La necesidad de profundizar el análisis en estos términos se hace aún más relevante cuando se observa que parece existir una relación directa entre las estrategias diferenciales que expresan los cambios en las transferencias y el lugar de la salud en la agenda local, medido en el porcentaje de gasto en salud existente en el presupuesto municipal.

Entre los casos que apuestan a aprovechar las reglas de juego del régimen de coparticipación, Malvinas Argentinas es el ejemplo paradigmático: su porcentaje de gasto en salud sobre el presupuesto total es del $35 \%$ y recibe la más alta transferencia per cápita de coparticipación en salud (total y por componentes no asociados al hospital), logrando financiar el $85 \%$ del gasto en salud a través de esta estrategia (m).

El municipio de Hurlingham, a pesar de haber "ganado" en las transferencias por coparticipación, está en los valores intermedios en transferencias por habitante (en consultas + establecimientos sin internación) y su gasto en salud es el $14,9 \%$ del gasto total municipal. Sin embargo, al final del período, logra financiar el $53 \%$ de su gasto en salud a través de las transferencias derivadas de la coparticipación en salud (n).
En el otro extremo, la estrategia de provincialización del hospital del municipio de Florencio Varela define un cuadro caracterizado por: valores intermedios para las transferencias por habitante, un porcentaje bajísimo de inversión en salud respecto del presupuesto municipal total y una cobertura a través de los recursos derivados de coparticipación en salud del $68 \%$.

\section{RECAPITULANDO... PARA VOLVER A ABRIR}

El recorrido realizado hasta aquí mostró los efectos de una descentralización (doméstica, esta vez) que, realizada sin definir previamente un modelo de salud que instituyera los servicios a ser garantizados y las responsabilidades de las distintas instancias gubernamentales, forzó la resolución -hacia fines de la década del ' 80 y a través de una reforma del régimen de coparticipación municipal- los problemas financieros que comenzaban a acumular los municipios, como consecuencia de la incorporación de los servicios transferidos.

Si bien el análisis realizado se basa en la utilización de medidas simples (como son, por ejemplo, el $r$ de Pearson y el análisis de diferencias porcentuales), fue posible observar que este sistema, originado en la necesidad de dar respuesta a los problemas de financiamiento y asociado a la distribución secundaria de la coparticipación, termina teniendo carácter devolutivo, en lugar de orientar la definición de políticas locales.

Es interesante rescatar que, dado el carácter cíclico de los recursos recibidos por los municipios en materia de coparticipación, se genera una dinámica en la cual los recursos descienden con las crisis y crecen cuando comienza a impactar positivamente la recuperación de la economía en la recaudación impositiva. Según la vinculación que establezca la política local con la variable financiera, este atributo de las transferencias puede tener distintos impactos en la naturaleza de la política local.

El análisis realizado en estas páginas mostró distintas situaciones en relación con estas reglas de juego en tanto incentivos o restricciones. Por una parte, la situación de casi veinte 
municipios que responden (con muy leves variaciones) a las tendencias agregadas. Por otra, la circunstancia particular de otros cuatro municipios que da cuenta de estrategias diferenciadas de incorporación de estas reglas de juego a la política local, sea por la vía de su aprovechamiento en la generación de ingresos o a través del alivio del gasto.

La mayor parte de los municipios parece haber apostado a no cambiar la estrategia o, probablemente, a no internalizar a esta como variable independiente de la definición de la política local, sino solo como una resultante. En estos casos, se limitaron a recibir las externalidades de un ciclo económico favorable que, considerando el incremento de los precios, apenas les permitiría recuperar los recursos previos a la crisis.

En contraste, en algunos otros casos, la estrategia explícita fue incorporar las reglas de juego como variable independiente en la definición de la política local. El municipio de Malvinas Argentinas constituye el caso más ejemplificador, donde el crecimiento de las camas y, posteriormente, la acreditación de complejidad de establecimientos, fue el dispositivo adoptado para alcanzar variaciones muy considerables en la participación que tenía respecto de la masa coparticipable al inicio del período y la que adquiere a posteriori. Desde el punto de vista político, estos esquemas, además de avanzar en la resolución de la brecha de financiamiento, permiten internalizar la totalidad de las derivaciones en los establecimientos bajo la órbita municipal, aumentando -paralelamente- los niveles de autonomía política y económica.

El tercer caso es el que representa el municipio de Florencio Varela, dándose una estrategia de reducción del gasto vía provincialización del hospital. Si bien Florencio Varela se torna menos vulnerable a la presencia/ausencia de transferencias provinciales, ve limitadas sus competencias para definir la estructura de su gasto en salud.
Es notable encontrar, más allá de las diferencias de apuesta, cierta convergencia con relación a lograr altos niveles de financiamiento externo del gasto realizado en el sector salud: tanto entre aquellas apuestas para aprovechar las condiciones del régimen de coparticipación expandiendo los servicios y la complejidad; como aquellas apuestas para reducir el gasto, provincializando el hospital.

Esta convergencia se refuerza aún más cuando vemos una gran dispersión en la situación de aquellos municipios que no produjeron cambios en el esquema de financiamiento: entre estos 20 municipios de la región podemos encontrar situaciones que oscilan entre el $19 \%$ y el $50 \%$ del financiamiento.

En este sentido, y conscientes de la necesidad de seguir buscando explicación a las diferencias, podría decirse que ambas estrategias están dinamizadas por la necesidad de cerrar la brecha de financiamiento del sector salud, originada por la descentralización de servicios y las alternativas posteriores de compensación por medio de transferencias, las cuales están muy determinadas por los ciclos de la economía. Estos, a su vez, impactan de manera directa en las características y magnitud de la demanda, tal como vimos en el análisis de la población sin cobertura de obras sociales y medicina privada.

Dadas las características que presenta el sistema de coparticipación en concepto de salud -que como vimos asigna los recursos de acuerdo a la estructura en salud disponible y a su utilizaciónel desafío se plantea en torno a los mecanismos que puedan encontrar los municipios para distribuir, en al actual esquema, los fondos de tal manera que tengan en cuenta verdaderamente las necesidades de bienes y servicios de salud y que impacten positivamente en la salud de la población. 


\section{NOTAS FINALES}

a. Este trabajo es parte de un proyecto de investigación colectivo más general titulado "Los sistemas de salud en el nivel local: mirando la gestión desde la atención de la salud de la población"; financiado por la Agencia Nacional de Promoción Científica y Tecnológica (ANPCyT), en el marco de los Proyectos de Investigación Científica y Tecnológica Orientados (PICTO), Universidad Nacional de General Sarmiento. Directora: Magdalena Chiara.

b. Este apartado se realizó en base a Chiara y Jiménez (1) y Chiara, Di Virgilio y Miraglia (2).

c. La coparticipación de impuestos es una forma de transferencia intergubernamental característica de los gobiernos multinivel, utilizada para compensar los desequilibrios territoriales y resolver la falta de correspondencia entre los recursos y los gastos. El caso analizado refiere a los mecanismos a través de los cuales se distribuyen los recursos desde la provincia hacia los municipios.

d. El crecimiento de los municipios del GBA respondió a distintos procesos de urbanización y se fueron conformando dos cordones concéntricos alrededor de la Ciudad Autónoma de Buenos Aires (CABA). El primer cordón está conformado por los municipios lindantes a la CABA, los que presentan la mayor densidad poblacional y tasas de crecimiento menor, mejor provisión de servicios urbanos y sociales y mejores indicadores de salud: Avellaneda, Gral. San Martín, Hurlingham, Ituzaingó, Lanús, Morón, San Isidro, Tres de Febrero y Vicente López. Estos se caracterizan por tener una población con menor proporción de niños y jóvenes, un aumento proporcional de personas mayores de 65 años, bajas tasas de natalidad, alta mortalidad general y un saldo migratorio negativo. El segundo cordón está constituido por los municipios de Almirante Brown, Berazategui, Esteban Echeverría, Ezeiza, Florencio Varela, José C. Paz, La Matanza, Lomas de Zamora, Malvinas Argentinas, Merlo, Moreno, Quilmes, San Fernando, San Miguel y Tigre. Aunque en los últimos años presentan una disminución en su tasa de crecimiento, tienen características muy distintas a las del primer cordón y a las del interior de la provincia. En términos de su estructura demográfica, se caracterizan por tener poblaciones más jóvenes, con una tasa de natalidad aún alta o estable y una mortalidad general baja y mayores niveles de población con necesidades básicas insatisfechas. A pesar de presentar algunos atributos comunes, se diferencian fuertemente -tal como veremos más adelante- en cuanto a los problemas que padece su población y en las condiciones que encuentran para dar respuesta a ellos desde la política pública. Un tercer grupo de municipios comienza a esbozar el denominado tercer cordón de la conurbación de la CABA. Se trata de los municipios de Escobar, Pilar y Presidente Perón, cuyas características comienzan a acercarse a aquellas del segundo cordón.

e. Desde 1998 la economía entra en una fase recesiva que termina en una crisis económica y financiera que se profundiza en el año 2001; dicho proceso culmina con la renuncia del presidente Fernando De la Rúa en diciembre de ese año. Se produce, entonces, una sucesión de presidentes y el 2 de enero de 2002 el Dr. Eduardo Duhalde, senador por la Provincia de Buenos Aires, es designado Presidente de la Nación.

f. En el sistema de coparticipación provincial, la distribución primaria es la parte de la recaudación total (que resulta de sumar los recursos provenientes de la coparticipación federal y los recursos propios) que la provincia va a distribuir entre los municipios. La distribución secundaria hace referencia a los criterios a través de los cuales se distribuyen esos recursos a cada uno de los municipios.

g. Algunos economistas observan que la cuestión conceptual de fondo se refiere a las externalidades que generan los servicios de salud, dado que además de beneficiar a la comunidad (a la que representan los municipios) probablemente beneficien a comunidades vecinas, resultando así clave el cuidado de dispositivos presupuestarios que internalicen las externalidades y la asunción por parte del gobierno provincial de aquellos servicios que comprometen a varios municipios (13).

h. Datos suministrados por la Subsecretaría de Planificación de la Salud del Ministerio de Salud de la Provincia de Buenos Aires.

i. En esta primera parte, se recupera el análisis realizado en Chiara, Di Virgilio y Miraglia (2).

j. El Municipio de Malvinas Argentinas se constituye en 1995, a partir de la división del ex partido de General Sarmiento en unidades territoriales menores. Malvinas Argentinas se instituyó sobre una territorialidad poco densificada en términos de instituciones y de actores, aspecto que permitió a las autoridades municipales modelar con una mayor libertad la institucionalidad en el nivel local.

k. Decimos "casi" en tanto estamos hablando del 2005. En el momento de redacción de este trabajo los efectores públicos de este municipio ya 
son contratados por el Ministerio de Salud de la provincia -y también por programas nacionalespara resolver prácticas de alta complejidad.

I. Según Belmartino (16) "Recibe la denominación de equipo de cambio, el grupo particular de agentes del Estado responsable de formular y promover el cambio de política".

m. La consolidación de la estrategia orientada al aprovechamiento de las reglas de juego encuentra un punto de apoyo en la continuidad de la autoridad política $y$, en particular, sanitaria. Intendente y Secretario de Salud Ilevan más de 10 años al frente del gobierno local y de su cartera sanitaria.

n. Los datos de porcentaje del gasto en salud financiado por transferencias de coparticipación fueron suministrados por la Subsecretaría de Planificación de la Salud del Ministerio de Salud de la Provincia de Buenos Aires, en el año 2006.

\section{REFERENCIAS BIBLIOGRÁFICAS}

1. Chiara $M$, Jiménez $C$. Relaciones intergubernamentales (RIGs) y política de salud en el nivel local. El caso del subsector público en el Gran Buenos Aires. Proveniente del XII Congreso Internacional del CLAD sobre la Reforma del Estado y de la Administración Pública; 30 de octubre al 1 de noviembre de 2007; Santo Domingo, República Dominicana. [Ponencia]. 2007.

2. Chiara $M$, Di Virgilio $M$, Miraglia $M$. Tensiones y dilemas en torno a la Gestión Local en Salud en el Gran Buenos Aires. En: Chiara M, Di Virgilio M, Medina A, Miraglia M, coordinadores. Gestión Local en Salud: conceptos y experiencias. Los Polvorines: Universidad Nacional de General Sarmiento; 2008.

3. Honorable Cámara de Senadores de la Provincia de Buenos Aires. Ley 7016. Regionalización Sanitaria de la Provincia de Buenos Aires. [En línea] Buenos Aires: Gobierno de la Provincia de Buenos Aires; 1965 [fecha de acceso 12 de diciembre de 2009]. URL disponible en: http://www.gob.gba.gov.ar/legislacion/legislacion/l $-7016 . h t m l$

4. Gobierno de la Provincia de Buenos Aires. Decreto 805/67. Regionalización sanitaria de la Provincia; reglamentación de la ley 7016. [En línea] Buenos Aires: Gobierno de la Provincia de Buenos Aires; 1967 [fecha de acceso 12 de diciembre de 2009]. URL disponible en: http://www.gob.gba.gov.ar/legislacion/legislacion/67-805.html

5. Instituto Nacional de Estadística y Censos. Censo Nacional de Población, Hogares y Viviendas 2001. [En línea] Buenos Aires: Indec; 2001 [fecha de acceso 12 de diciembre de 2009]. URL disponible en: http://www.indec.gov.ar/webcenso/index.asp
6. Chiara M, Di Virgilio M. Municipios y gestión social. De los escritos del Banco Mundial a las calles del Gran Buenos Aires. Los Polvorines: Universidad Nacional de General Sarmiento; 2005.

7. Jordana J. Relaciones intergubernamentales y descentralización en América Latina: una perspectiva institucional. Washington DC: INDES, Unión Europea; 2001. (Serie Documentos de Trabajo I-22UE).

8. Honorable Cámara de Senadores de la Provincia de Buenos Aires. Ley 10820. [En línea] Buenos Aires: Gobierno de la Provincia de Buenos Aires; 1989 [fecha de acceso 12 de diciembre de 2009]. URL disponible en: http://www.gob.gba.gov.ar/legislacion/legislacion/l-10820.html

9. Honorable Cámara de Senadores de la Provincia de Buenos Aires. Ley 10559. [En línea] Buenos Aires: Gobierno de la Provincia de Buenos Aires; 1987 [fecha de acceso 12 de diciembre de 2009]. URL disponible en: http://www.gob.gba.gov.ar/legislacion/legislacion/l-10559.html

10. Iarussi M. La coparticipación en la provincia de Buenos Aires y el sector salud. En: Chiara M, Di Virgilio M, Medina A, Miraglia M, coordinadores. Gestión Local en Salud: conceptos y experiencias. Los Polvorines: Universidad Nacional de General Sarmiento; 2008.

11. Barbieri N. Política fiscal y política sanitaria: tensiones evidentes a partir de los criterios de reparto de la masa coparticipable y los métodos de determinación de transferencia. Salud Colectiva. 2007;3(1):49-61.

12. Ministerio de Economía de la Provincia de Buenos Aires. Estado de situación y propuesta de reforma del sistema médico asistencial público de 
la Provincia de Buenos Aires. [En línea] Cuadernos de Economía. 2002; (63): 1-88 [fecha de acceso 12 de diciembre de 2009]. URL disponible en: http://www.ec.gba.gov.ar/Prensa/Archivos/Cuader no63.pdf

13. Garriga M, Sanguinetti P. Coparticipación a Municipalidades de la Provincia de Buenos Aires. Cuadernos de Economía. 1995;(10):1-88.

14. Instituto Nacional de Estadísticas y Censos. Sistemas de índices de precios mayoristas [En línea] Buenos Aires: INDEC; 2005 [fecha de acceso 2 de agosto de 2006] URL disponible en: http://www.indec.mecon.gov.ar/principal.asp?id _tema $=732$

15. Subsecretaría de Planificación de la Salud. Establecimientos con y sin internación y promedio de camas disponibles de dependencia provincial, municipal y nacional por región sanitaria. Provincia de Buenos Aires. [En línea] Provincia de Buenos Aires: Ministerio de Salud; 2005 [fecha de acceso 2 de febrero de 2010]. URL disponible en: http://www.ms.gba.gov.ar/estadisticas/establecimientos.html

16. Belmartino S. La gestión local de atención médica abordada como objeto de investigación. En: Chiara M, Di Virgilio M, Medina A, Miraglia M, coordinadores. Gestión Local en Salud: conceptos y experiencias. Los Polvorines: Universidad Nacional de General Sarmiento; 2008.

17. Subsecretaría de Planificación de la Salud. Estadísticas vitales. [En línea] Provincia de Buenos Aires: Ministerio de Salud; 2005 [fecha de acceso 2 de febrero de 2010] URL disponible en: http://www.ms.gba.gov.ar/estadisticas/estadisticas_vitales.html

\section{FORMA DE CITAR}

Chiara M, Di Virgilio M, Ariovich A. La coparticipación provincial por salud en el Gran Buenos Aires: Reflexiones en torno a la política local (2000-2005). Salud Colectiva. 2010;6(1):74-64.

Recibido el 23de marzo de 2009

Versión final presentada el 15 de octubre de 2009

Aprobado el 30 de noviembre de 2009 